\title{
MGMT promoter methylation in serum and cerebrospinal fluid as a tumor-specific biomarker of glioma
}

\author{
ZHENG WANG ${ }^{*}$, WEI JIANG ${ }^{*}$, YAHONG WANG, YANG GUO, \\ ZHENG CONG, FANGFANG DU and BIN SONG \\ Department of Radiation Oncology, Tianjin Huanhu Hospital, Tianjin 300060, P.R. China
}

Received March 6, 2015; Accepted May 5, 2015

DOI: $10.3892 /$ br.2015.462

\begin{abstract}
O}^{6}$-methylguanine-DNA methyltransferase $(M G M T)$ promoter methylation is a conventional technique to predict the prognosis or individualized treatment of glioma in tumor tissue following surgery or biopsy. However, the technique cannot be applied in those glioma patients with concomitant neurological dysfunctions or advanced age. The present study aimed to find a new minimally invasive and efficient alternative method for the detection of $M G M T$ promoter methylation. The expression of $M G M T$ promoter methylation was assessed in peripheral blood and cerebrospinal fluid (CSF), and compared to the corresponding tumor tissue from glioma patients. The 89 patients in the study [32 World Health Organization (WHO) grade II, 19 WHO grade III and 38 WHO grade IV) were pathologically-diagnosed glioma and received radiation therapy following sample collection. The resected glioma tumor tissue (89), corresponding serum (89) and CSF (78) samples were collected for the detection of $M G M T$ promoter methylation using methylation-specific polymerase chain reaction. The sensitivity and specificity of detecting MGMT promoter methylation in CSF and serum were compared. Among the tumor tissue samples, 51/89 (57.3\%) showed MGMT promoter methylation. The specificity of the detection in the CSF and serum samples reached $100 \%$. The sensitivity of $M G M T$ promoter methylation detection
\end{abstract}

Correspondence to: Dr Wei Jiang, Department of Radiation Oncology, Tianjin Huanhu Hospital, 122 Qixiangtai Road, Hexi, Tianjin 300060, P.R. China

E-mail: weijianghh@163.com

*Contributed equally

Abbreviations: MGMT, $\mathrm{O}^{6}$-methylguanine-DNA methyltransferase; CSF, cerebrospinal fluid; PCR, polymerase chain reaction; TMZ, temozolomide; RTOG, Radiation Therapy Oncology Group; WHO, World Health Organization; MeDIP, methylated DNA immunoprecipitation

Key words: $\mathrm{O}^{6}$-methylguanine-DNA methyltransferase, glioma, circulating DNA, cerebrospinal fluid, tumor-specific molecular marker in CSF and serum were 26/40 (65.0\%) and 19/51 (37.3\%), respectively $(\mathrm{P}<0.05)$. In the WHO II, III and IV subgroups, the sensitivities of $M G M T$ promoter methylation detection using CSF were 8/12 (66.7\%), 11/18 (61.1\%) and 7/10 (70.0\%), respectively, which were significantly higher than the sensitivities using serum $(7 / 21,33.3 \% ; 7 / 19,36.8 \%$; and 5/11, 45.5\%, respectively $\mathrm{P}<0.05)$. Among patients with residual postoperative tumors, the sensitivities of detecting $M G M T$ promoter methylation using CSF and serum were 18/25 (72.0\%) and $10 / 24(41.7 \%)$, respectively, both of which were significantly higher than the corresponding values for patients without residual tumors $(8 / 15,53.3 \%$ and $6 / 19,31.6 \%$, respectively; $\mathrm{P}<0.05)$. The detection of $M G M T$ promoter methylation in CSF specimens shows higher sensitivity compared to the serum for glioma patients. Assessment of MGMT promoter methylation in CSF may provide a promising clinical methodology for early diagnosis, individual treatment, monitoring of recurrence and prognosis for glioma patients.

\section{Introduction}

$\mathrm{O}^{6}$-methylguanine-DNA methyltransferase $(M G M T)$ is a DNA repair protein that is closely associated with alkylating agents of glioma cells $(1,2)$. In 2009, a large prospective phase III clinical study conducted by the European Organization for Research and Treatment of Cancer and the National Cancer Institute of Canada confirmed that newly diagnosed patients with glioblastoma accompanied by $M G M T$ gene promoter methylation could benefit from radiotherapy with concomitant adjuvant temozolomide (TMZ) treatment (3). Subsequently, at the 2011 American Society of Clinical Oncology annual meeting, the Radiation Therapy Oncology Group (RTOG) reported the results of a phase III randomized controlled trial (RTOG 0525), which included 833 cases of malignant glioma; the survival time of patients with MGMT gene promoter methylation was significantly longer than that of patients without $M G M T$ gene promoter methylation after receiving standard TMZ treatment (4). These findings support an independent role for $M G M T$ gene promoter methylation in predicting prognosis. NOA-08, Nordic and a few other recent large clinical trials have also confirmed that $M G M T$ promoter methylation can independently predict the prognosis of elderly patients with newly diagnosed glioblastoma after receiving TMZ treatment (5-8). Thus, the use of MGMT and 
other indicators for molecular typing of malignant glioma and selection of standardized, individualized treatment regimens are crucial for controlling the progression of malignant glioma and predicting its prognosis.

Currently, postoperative or biopsy specimens are typically used for testing $M G M T$ promoter methylation in glioma patients. However, the majority of glioma patients also have varying degrees of concomitant neurological dysfunctions, which manifest as acute or chronic systemic diseases. The associated neurological dysfunctions, as well as the advanced age of the population that commonly presents with glioma, restricts tissue sample collection by surgery or biopsy, thereby affecting the clinical diagnosis and design of individualized treatment plans and ultimately compromising the treatment effect. Therefore, finding a new non-invasive and efficient alternative method for the detection of $M G M T$ promoter methylation is important.

In the blood circulation and body fluid of cancer patients, the level of free DNA is significantly higher compared to normal controls and circulating tumor-specific molecular markers have been used to detect a variety of tumors (9). In 2010, Lavon et al (10) performed a paired examination of $M G M T$ promoter methylation in tissues and the corresponding serum of patients with different grades of glioma [World Health Organization (WHO) grade II-IV]. The overall sensitivity of the test for $M G M T$ gene promoter methylation using DNA in the serum was $51 \%$ and the specificity was $100 \%$. Due to the existence of the blood-brain barrier as a unique physiological barrier, cerebrospinal fluid (CSF) should in theory contain higher levels of circulating tumor DNA, particularly for primary tumors of the nervous system, such as gliomas. Consequently, we hypothesized that CSF should be more suitable for detecting tumor-associated molecules than serum. In the present study, to analyze the applicability of detecting $M G M T$ promoter methylation using CSF, MGMT promoter methylation was assessed using tumor tissues and serum in comparison to corresponding CSF samples. The results suggest that assessment of $M G M T$ promoter methylation in CSF may provide a promising clinical strategy for early diagnosis, design of individualized treatment plans, monitoring of recurrence or prognosis of glioma patients.

\section{Materials and methods}

Sample collection. A total of 89 patients were included in the study, and all were pathologically-diagnosed glioma and received radiation therapy at the Department of Radiation Oncology of Tianjin Huanhu Hospital (Tianjin, China) between July 2012 and December 2013. Tumor tissue samples, the corresponding serum and CSF specimens were collected from each patient for the detection of $M G M T$ promoter methylation. Grade of glioma was conducted according to the classification criteria for tumors of the central nervous system published by WHO (11). There were 32 cases of WHO II, 19 of WHO III and 38 of WHO IV glioma. The characteristics of the patients are shown in Table I. All the blood and CSF samples of the patients corresponding to the tumor samples were collected postoperatively and prior to radiotherapy, and were processed immediately following sample collection. All the CSF samples were achieved by lumbar puncture and the blood samples
Table I. Characteristics of patients in the study $(\mathrm{n}=89)$.

\begin{tabular}{lc}
\hline Characteristics & Patients \\
\hline Age, median years (range) & $47(12-79)$ \\
Gender, $\mathrm{n}(\%)$ & \\
Male & $53(59.6)$ \\
Female & $36(40.4)$ \\
Histology, $\mathrm{n}(\%)$ & \\
Grade II & $32(36.0)$ \\
Grade III & $19(21.3)$ \\
Grade IV & $38(42.7)$ \\
Surgical extent, n (\%) & \\
GTR & $38(42.7)$ \\
STR & $51(57.3)$
\end{tabular}

GTR, gross total resection; STR, subtotal resection.

were collected at the same time. The median time interval between DNA extraction from the blood and CSF samples and the surgery was 19 days (range, 11-42 days). Blood and CSF samples were also collected from 20 healthy volunteers as the control. The study was approved by the Research Ethics Committee of Tianjin Huanhu Hospital. Informed consent was obtained from all patients.

\section{DNA extraction}

Tissue DNA extraction. The classic phenol-chloroform extraction method was applied. For fresh tissue, the TIANamp Genomic DNA kit (Tiangen Biotech, Beijing, China) was used to extract genomic DNA. For paraffin sections, the samples were dewaxed prior to being subjected to genomic DNA extraction using the TIANamp Genomic DNA kit.

Cerebrospinal fluid DNA extraction. CSF samples (4-5 ml) were centrifuged and the supernatant was discarded. Genomic DNA was isolated from the sediment using the TIANamp Genomic DNA kit.

DNA extraction from peripheral blood. Following collection, peripheral blood samples were immediately subjected to centrifugation; the serum was isolated and stored at $-80^{\circ} \mathrm{C}$. The Qiagen Blood DNA kit (Qiagen, Hilden, Germany) was used for the extraction of free DNA. For the control group, $\mathrm{X}$ denotes whole blood DNA of a normal human subject (unmethylated control) and X-Sss I denotes the whole blood DNA of the same subject, modified by Sss I (New England BioLabs, Ltd., Hitchin, UK) following the manufacturer's instructions (methylated control).

Sulfite modification and detection of MGMT gene methylation. Purification of genomic DNA was performed using the Wizard DNA purification kit (Promega Corp., Madison, WI, USA) following the manufacturer's instructions. For the detection of $M G M T$ gene methylation, the methylation-specific polymerase chain reaction (PCR) method was applied and the primers were as follows: $M G M T$ forward, 5'-CAGGACCGGGATTC TCACTA-3' and reverse, 5'-CCAAATGGCCCG TACCTT-3'; and amplification conditions were as described (12). The PCR 
products were subjected to denaturation for high-performance liquid chromatography analysis with the following steps: PCR products were loaded onto a DNASep ${ }^{\circledR}$ nucleic acid analysis column (Transgenomic, Inc., Omaha, NE, USA) and were subjected to multi-fragment separation using the double-stranded multiple fragments mode of the Transgenomic WAVE System (fragment sizes, $20-100 \mathrm{bp}$; temperature, $50^{\circ} \mathrm{C}$; flow rate, $0.9 \mathrm{ml} / \mathrm{min}$; and ultraviolet detection wavelength, $260 \mathrm{~nm}$ ). Navigator software was used for analysis of the results. Peripheral blood lymphocyte DNA of normal subjects was used as a negative methylation control and peripheral blood lymphocyte DNA treated with the methylation enzyme Sss I was used as a positive methylation control. For the blank control, distilled water was used instead of the DNA template.

Statistical analysis. Concordance between the biomarker (MGMT promoter methylation) in tumor tissue, serum and CSF was assessed by the $\kappa$-measure of agreement. Association was tested using the $\chi^{2}$ test. $\mathrm{P}<0.05$ was considered to indicate a statistically significant difference. Statistical analysis was performed in the SPSS software package, version 16.0 (SPSS, Inc., Chicago, IL, USA).

\section{Results}

Sample collection. To compare the sensitivity and specificity of assessing MGMT promoter methylation in the blood and CSF of glioma patients as an indicator of disease, 256 samples were collected from the 89 glioma patients (including tumor tissue and matched blood and/or CSF samples). Blood samples matching the tumor tissues were collected from all the patients and matched CSF samples were collected from all except 11 patients (9 with grade II and 2 with grade III). Blood and matched CSF samples were also collected from 20 healthy volunteers as the control and all these samples tested negative for $M G M T$ promoter methylation.

MGMT promoter methylation detection. Table II provides the results of $M G M T$ promoter methylation detection for the patient samples. Among the tumor tissue samples, 51/89 (57.3\%) showed $M G M T$ promoter methylation; further subgroup analysis revealed that the proportions of $M G M T$ promoter methylation in the tumor tissue sample of WHO II, III and IV glioma were $21 / 32(65.6 \%), 11 / 19(57.9 \%)$ and 19/38 (50.0\%), respectively, which did not differ significantly between the groups (Table II). Of note, the proportion of MGMT promoter methylation positivity in tumor tissues that had an oligodendroglioma component (20/29, 69.0\%) was significantly higher than those that did not (31/60, 51.7\%), $\mathrm{P}<0.05$. This result is consistent with previous reports suggesting that $M G M T$ promoter methylation may be associated with the occurrence of oligodendroglioma. Among all the CSF and blood samples, 45 tested positive for $M G M T$ promoter methylation, including 19/89 (21.3\%) of the blood samples and 26/78 (33.3\%) of the CSF samples (Table II). These results suggest that assessment of $M G M T$ promoter methylation may be more sensitive for CSF than for blood serum.

Sensitivity and specificity testing. To further assess the sensitivity and specificity of testing $M G M T$ promoter methylation in serum versus CSF, the specific results were analyzed for
Table II. Detection of MGMT promoter methylation using different samples.

\begin{tabular}{lccr}
\hline & \multicolumn{3}{c}{ Samples, $\mathrm{n}(\%)$} \\
\cline { 2 - 4 } Grade & Tumor tissue, $\mathrm{n}=89$ & Serum, $\mathrm{n}=89$ & $\mathrm{CSF}, \mathrm{n}=78$ \\
\hline II & $21(65.6)$ & $7(21.9)$ & $8(34.8)$ \\
III & $11(57.9)$ & $5(26.3)$ & $7(38.9)$ \\
IV & $19(50.0)$ & $7(18.4)$ & $11(29.7)$ \\
\hline
\end{tabular}

$M G M T, \mathrm{O}^{6}$-methylguanine-DNA methyltransferase; CSF, cerebrospinal fluid.

Table III. Sensitivity and specificity of $M G M T$ promoter methylation detection for serum and CSF samples with positive tests for corresponding tumor tissue.

\begin{tabular}{lccccc}
\hline & \multicolumn{2}{c}{ Serum, $\mathrm{n}=51$} & & \multicolumn{2}{c}{ CSF, $\mathrm{n}=40$} \\
\cline { 2 - 3 } \cline { 5 - 6 } Variables & $\mathrm{n}(\%)$ & P-value & $\mathrm{n}(\%)$ & P-value \\
\hline Grade & & & & \\
II & $7(33.3)$ & $>0.05$ & & $8(66.7)$ & $>0.05$ \\
III & $5(45.5)$ & & $7(70.0)$ & \\
IV & $7(36.8)$ & & $11(61.1)$ & \\
Histology & & & & \\
Oligo $^{+}$ & $8(40.0)$ & $>0.05$ & $8(66.7)$ & $>0.05$ \\
Oligo $^{-}$ & $11(35.4)$ & & $18(64.2)$ & \\
\hline
\end{tabular}

MGMT, $\mathrm{O}^{6}$-methylguanine-DNAmethyltransferase; CSF, cerebrospinal fluid; oligo, oligodendroglioma.

the 51 tumor tissues that tested positive for $M G M T$ promoter methylation. Of these 51 positive samples, 51 matched serum samples and 40 matched CSF samples were collected. The overall sensitivity of $M G M T$ promoter methylation detection was higher for CSF (26/40, 65.0\%) than for serum (19/51, $37.3 \%$ ) ( $\mathrm{P}<0.05)$ (Table III). Notably, all 19 serum samples that tested positive for $M G M T$ promoter methylation for the blood serum also tested positive for $M G M T$ promoter methylation for the CSF. Subgroup analysis revealed that the sensitivity using CSF in WHO II, III and IV glioma patients (8/12, 66.7\%; $11 / 18,61.1 \%$ and $7 / 10,70.0 \%$ respectively) was significantly higher than the corresponding values obtained using serum $(7 / 21,33.3 \% ; 7 / 19,36.8 \%$; and $5 / 11,45.5 \%$, respectively) $(\mathrm{P}<0.05)$, thus verifying the uniformly higher sensitivity of the CSF samples. Furthermore, the increased sensitivity of CSF $(8 / 12,66.7 \%$ or $18 / 28,64.2 \%)$ versus serum $(8 / 20,40.0 \%$ or $11 / 31,35.4 \%$ ) was also conserved regardless of whether or not the tumor tissues had an oligodendroglioma component (oligo $^{+}$or oligo ${ }^{-}$, Table III; $\mathrm{P}<0.05$ ). These results suggest that using the CSF of patients with tumors of the nervous system may be advantageous over using serum for free DNA detection.

To determine the specificity of detection, whether the matched blood/CSF samples that tested positive for MGMT promoter methylation also tested positive for the tumor tissues 
Table IV. Detection of MGMT promoter methylation among patients with different postoperative residual tumors.

\begin{tabular}{lccc}
\hline Samples & GTR,n $(\%)$ & STR,n $(\%)$ & P-value \\
\hline Serum & $6(31.6)$ & $10(41.7)$ & $<0.05$ \\
CSF & $8(53.3)$ & $18(72.0)$ & \\
\hline
\end{tabular}

MGMT, $\mathrm{O}^{6}$-methylguanine-DNA methyltransferase; GTR, gross total resection; STR, subtotal resection; CSF, cerebrospinal fluid.

Table V. Expression of $M G M T$ promoter methylation between different grades and the postoperative residual tumors.

\begin{tabular}{lccc}
\hline & \multicolumn{3}{c}{ WHO Grade } \\
\cline { 2 - 4 } Samples & II, n (\%) & III, n (\%) & IV, n (\%) \\
\hline Serum & & & \\
GTR & $1(16.7)$ & $2(33.3)$ & $3(42.9)$ \\
STR & $6(50.0)$ & NS & $4(33.3)$ \\
CSF & & & \\
GTR & $1(33.3)$ & $3(60.0)$ & $4(57.1)$ \\
STR & $7(77.8)$ & $4(80.0)$ & $7(63.6)$ \\
\hline
\end{tabular}

MGMT, $\mathrm{O}^{6}$-methylguanine-DNAmethyltransferase; WHO, World Health Organization; GTR, gross total resection; STR, subtotal resection; NS, no samples were available for this subgroup; CSF, cerebrospinal fluid.

was calculated. For serum and CSF, the specificity reached $100 \%$; none of the tumor tissue samples that tested negative for MGMT promoter methylation had corresponding CSF or blood samples that tested positive. Additionally, none of the CSF and blood samples collected from the 20 healthy subjects in the control group tested positive for MGMT promoter methylation, providing further support for the specificity (data not shown).

Association of tumor occurrence and MGMT promoter methylation. To further assess the utility of this approach for patients of different post-operative outcomes, the association was examined between the occurrence of residual tumor and the detection of $M G M T$ promoter methylation using serum and CSF (Tables IV and V). All the magnetic resonance images used to analyze postoperative residual tumors were collected within one week from the collection of blood and CSF samples. Patients without residual tumors had sensitivities for serum and CSF samples of 6/19 (31.6\%) and 8/15 (53.3\%), respectively; while patients with residual tumors had sensitivities of $10 / 24(41.7 \%)$ and 18/25 (72\%), respectively. There was a similar trend of greater sensitivity for the CSF versus the serum among subgroups with different tumor grades (Tables IV and V). Of note, the detection of $M G M T$ promoter methylation for the patients with WHO III and WHO IV tumors was higher than for patients with WHO II tumors for the serum and CSF of patients without residual tumors, although this trend was not conserved for patients with residual tumors. Overall, these results suggest that the occurrence of $M G M T$ promoter methylation is greater for patients with residual tumors, but that CSF is more sensitive than blood for detecting the $M G M T$ promoter methylation for either patient population $(\mathrm{P}<0.05)$.

\section{Discussion}

$M G M T$-mediated resistance to alkylating agents is now considered as the key factor compromising postoperative adjuvant treatment for glioma patients. Therefore, the detection of MGMT methylation is of great clinical value in selecting individualized treatment plans and assessing prognosis for patients with glioma. Currently, the main source of samples used for detecting $M G M T$ promoter methylation is tumor tissue obtained from surgery or biopsy; however, in clinical practice a large number of glioma patients are unable to undergo surgery. Therefore, the establishment of a simple, minimally invasive method for detecting $M G M T$ methylation is required. Circulating DNA is present in body fluids (CSF and blood) and can be used to detect certain cancer gene mutations of primary tumors $(13,14)$. Consequently, this method has been successfully applied in the diagnosis and treatment of numerous types of cancer (breast, prostate, liver and lung cancer) (15-19). However, there are few reports on its application for tumors of the nervous systems. Lavon et al (10) used methylated DNA immunoprecipitation (MeDIP) to detect the methylation of promoters of $M G M T$ and other cancer-related genes using tumor samples and matched serum samples collected from 70 patients with different levels of glioma. Although the circulating DNA in serum exhibited genetic alterations highly consistent with that of the primary tumors, the sensitivity of detection of this method was only $\sim 50 \%$. The content of circulating DNA in CSF is higher than that in serum, due in part to the blood-brain barrier, which prevents clearing of circulating DNA by the liver (20). Therefore, we postulated that the sensitivity may be increased by assessing CSF rather than blood.

To determine whether the use of CSF may increase the sensitivity of $M G M T$ promoter methylation detection, tumor tissue samples and corresponding blood and CSF samples were examined by methylation-specific PCR in the present study. The results demonstrate that CSF is superior to serum in DNA detection of tumors of the nervous system, with overall detection of $65.0 \%$ for CSF samples versus $37.3 \%$ for serum samples. The greater sensitivity was retained across subsets of patients at different tumor grades. The sensitivity of detecting MGMT methylation in serum in the present study $(37.3 \%)$ is consistent with certain other studies reporting patients of other solid tumors $(19,21)$, but is lower than that reported by Lavon et al (10) in gliomas (51.0\%), and this may be explained by the studies designed in different populations or different detection methods applied.

In the present study, CSF and serum samples were collected following the surgery and subsequent to the start of the radiotherapy, and the median time interval between the sample collection and the surgery was 19 days (11-42 days) (Table I). Among the 38 patients who had no residual tumors, as assessed by magnetic resonance imaging, the sensitivity of detecting $M G M T$ promoter methylation using CSF and serum was 
$8 / 15(53.3 \%)$ and 6/19 (31.6\%), respectively. For patients who had positive tissue sample results but did not show residual tumors, positive $M G M T$ promoter methylation test results could still be obtained using CSF and blood samples. This result is consistent with another report (10). Associated studies have shown that the half-life of circulating tumor DNA is only $\sim 2 \mathrm{~h}$ (13), and therefore, the above results suggest that continued release of circulating tumor DNA into the CSF and serum is sustained for glioma patients, even without residual tumors being detected by postoperative imaging. This phenomenon may be associated with the invasive nature of glioma growth; in addition to the solid tumors that can be detected using traditional imaging techniques, a wide range of subclinical lesions cannot be detected with traditional imaging. The present study also revealed that the sensitivity of detecting MGMT promoter methylation for the 'residual tumor' group $(72.0 \%$ for CSF; $41.7 \%$ for blood) was significantly higher than for the "no residual tumor' group (53.3\% for CSF; $31.6 \%$ for blood), which highlights a potential correlation between $M G M T$ positivity and tumor status $(17,22,23)$. Of note, for the group with no observable residual postoperative tumor, the sensitivity of detecting MGMT promoter methylation in high-grade glioma (WHO III and IV) was significantly higher compared to low-grade glioma (WHO II), yet this difference was not observed in the group with residual tumors (Table IV). These findings may provide additional support for a more prominent role for the subclinical lesions associated with higher tumor grades.

Although CSF specimens have been shown to offer higher sensitivity than serum, the average detection sensitivity of $65.0 \%$ suggests that further improvement on the methodology could be achieved. The selection of an appropriate target DNA fragment is a key factor affecting the sensitivity of the test. Although the majority of studies have favored the detection of low-molecular-weight DNA (10), the majority of these studies have targeted tumors outside of the nervous system. Further investigation is required to determine the optimal fragment length for DNA assessment in the nervous system. Additionally, the sensitivity of $M G M T$ promoter methylation detection could possibly be improved via the use of quantitative methylation methods. A large number of emerging technologies have utilized quantitative methylation analysis for tumor samples and other body fluid samples (24-28). Liu et al (28) successfully applied MeDIP to detect the methylation of a variety of tumor-specific markers, including MGMT, using tumor tissue, matched serum and CSF samples collected from patients with malignant glioma; the positive rates of $M G M T$ promoter methylation in tumor tissue, serum and CSF were 97.0, 71.2 and 78.8\%, respectively. These values cannot be directly compared to the present results due to differences in the populations and the methodology; however, the generally higher sensitivity in the latter study suggests the possibility that quantitative methylation detection techniques may further improve the detection sensitivity. Finally, to achieve highly efficient and accurate early diagnosis, additional tumor-specific molecular markers closely associated with the diagnosis and prognosis of glioma patients may be included for future studies.

In conclusion, $M G M T$ promoter methylation of circulating DNA in serum and CSF was successfully applied to patients with different levels of glioma. The results show a good prospect of using CSF specimens for the detection of circulating DNA in tumors of the nervous system. In the future, large-scale clinical trials are required to validate the clinical value of detecting circulating DNA using CSF samples for the early diagnosis, selection of individualized treatment plan, monitoring of recurrence and prognosis of glioma patients.

\section{Acknowledgements}

The present study was supported by the Science and Technology Foundation of Tianjin Health Bureau (grant no. 2012KR10).

\section{References}

1. Friedman HS, McLendon RE, Kerby T, Dugan M, Bigner SH, Henry AJ, Ashley DM, Krischer J, Lovell S, Rasheed K, et al: DNA mismatch repair and O6-alkylguanine-DNA alkyltransferase analysis and response to Temodal in newly diagnosed malignant glioma. J Clin Oncol 16: 3851-3857, 1998.

2. Esteller M, Garcia-Foncillas J, Andion E, Goodman SN, Hidalgo OF, Vanaclocha V, Baylin SB and Herman JG: Inactivation of the DNA-repair gene MGMT and the clinical response of gliomas to alkylating agents. N Engl J Med 343: $1350-1354,2000$

3. Stupp R, Hegi ME, Mason WP, van den Bent MJ, Taphoorn MJ, Janzer RC, Ludwin SK, Allgeier A, Fisher B, Belanger K, et al; European Organisation for Research and Treatment of Cancer Brain Tumour and Radiation Oncology Groups; National Cancer Institute of Canada Clinical Trials Group: Effects of radiotherapy with concomitant and adjuvant temozolomide versus radiotherapy alone on survival in glioblastoma in a randomised phase III study: 5-year analysis of the EORTC-NCIC trial. Lancet Oncol 10: 459-466, 2009.

4. Weiler M, Hartmann C, Wiewrodt D, Herrlinger U, Gorlia T, Bähr O, Meyermann R, Bamberg M, Tatagiba M, von Deimling A, et al: Chemoradiotherapy of newly diagnosed glioblastoma with intensified temozolomide. Int J Radiat Oncol Biol Phys 77: 670-676, 2010.

5. Reifenberger G, Hentschel B, Felsberg J, Schackert G, Simon M, Schnell O, Westphal M, Wick W, Pietsch T, Loeffler M, et al; German Glioma Network: Predictive impact of MGMT promoter methylation in glioblastoma of the elderly. Int J Cancer 131: 1342-1350, 2012.

6. Gállego Pérez-Larraya J, Ducray F, Chinot O, Catry-Thomas I, Taillandier L, Guillamo JS, Campello C, Monjour A, Cartalat-Carel S, Barrie M, et al: Temozolomide in elderly patients with newly diagnosed glioblastoma and poor performance status: An ANOCEF phase II trial. J Clin Oncol 29: 3050-3055, 2011.

7. Wick W, Platten M, Meisner C, Felsberg J, Tabatabai G, Simon M, Nikkhah G, Papsdorf K, Steinbach JP, Sabel M, et al; NOA-08 Study Group of Neuro-oncology Working Group (NOA) of German Cancer Society: Temozolomide chemotherapy alone versus radiotherapy alone for malignant astrocytoma in the elderly: The NOA-08 randomised, phase 3 trial. Lancet Oncol 13: 707-715, 2012.

8. Malmström A, Grønberg BH, Marosi C, Stupp R, Frappaz D, Schultz H, Abacioglu U, Tavelin B, Lhermitte B, Hegi ME, et al; Nordic Clinical Brain Tumour Study Group (NCBTSG): Temozolomide versus standard 6-week radiotherapy versus hypofractionated radiotherapy in patients older than 60 years with glioblastoma: The Nordic randomised, phase 3 trial. Lancet Oncol 13: 916-926, 2012.

9. Schwarzenbach H, Hoon DS and Pantel K: Cell-free nucleic acids as biomarkers in cancer patients. Nat Rev Cancer 11: 426-437, 2011

10. Lavon I, Refael M, Zelikovitch B, Shalom E and Siegal T: Serum DNA can define tumor-specific genetic and epigenetic markers in gliomas of various grades. Neuro Oncol 12: 173-180, 2010.

11. Louis DN, Ohgaki H, Wiestler OD, Cavenee WK, Burger PC, Jouvet A, Scheithauer BW and Kleihues P: The 2007 WHO classification of tumours of the central nervous system. Acta Neuropathol 114: 97-109, 2007.

12. Esteller M, Hamilton SR, Burger PC, Baylin SB and Herman JG: Inactivation of the DNA repair gene O6-methylguanine-DNA methyltransferase by promoter hypermethylation is a common event in primary human neoplasia. Cancer Res 59: 793-797, 1999. 
13. Diehl F, Schmidt K, Choti MA, Romans K, Goodman S, Li M, Thornton K, Agrawal N, Sokoll L, Szabo SA, et al: Circulating mutant DNA to assess tumor dynamics. Nat Med 14: 985-990, 2008.

14. Yoon KA, Park S, Lee SH, Kim JH and Lee JS: Comparison of circulating plasma DNA levels between lung cancer patients and healthy controls. J Mol Diagn 11: 182-185, 2009.

15. Chan KC and Lo YM: Circulating tumour-derived nucleic acids in cancer patients: Potential applications as tumour markers. Br J Cancer 96: 681-685, 2007.

16. Chan KC, Lai PB, Mok TS, Chan HL, Ding C, Yeung SW and Lo YM: Quantitative analysis of circulating methylated DNA as a biomarker for hepatocellular carcinoma. Clin Chem 54 1528-1536, 2008.

17. Usadel H, Brabender J, Danenberg KD, Jerónimo C, Harden S, Engles J, Danenberg PV, Yang S and Sidransky D: Quantitative adenomatous polyposis coli promoter methylation analysis in tumor tissue, serum, and plasma DNA of patients with lung cancer. Cancer Res 62: 371-375, 2002.

18. Umetani N, Giuliano AE, Hiramatsu SH, Amersi F, Nakagawa T, Martino S and Hoon DS: Prediction of breast tumor progression by integrity of free circulating DNA in serum. J Clin Oncol 24: 4270-4276, 2006

19. Sunami E, Shinozaki M, Higano CS, Wollman R, Dorff TB Tucker SJ, Martinez SR, Mizuno R, Singer FR and Hoon DS: Multimarker circulating DNA assay for assessing blood of prostate cancer patients. Clin Chem 55: 559-567, 2009.

20. Hisazumi J, Kobayashi N, Nishikawa M and Takakura Y: Significant role of liver sinusoidal endothelial cells in hepatic uptake and degradation of naked plasmid DNA after intravenous injection. Pharm Res 21: 1223-1228, 2004.

21. Tsang JC and Lo YM: Circulating nucleic acids in plasma/serum. Pathology 39: 197-207, 2007.
22. Hoque MO, Topaloglu O, Begum S, Henrique R, Rosenbaum E, Van Criekinge W, Westra WH and Sidransky D: Quantitative methylation-specific polymerase chain reaction gene patterns in urine sediment distinguish prostate cancer patients from control subjects. J Clin Oncol 23: 6569-6575, 2005.

23. Fackler MJ, Malone K, Zhang Z, Schilling E, Garrett-Mayer E, Swift-Scanlan T, Lange J, Nayar R, Davidson NE, Khan SA, et al: Quantitative multiplex methylation-specific PCR analysis doubles detection of tumor cells in breast ductal fluid. Clin Cancer Res 12: 3306-3310, 2006.

24. Weber M, Davies JJ, Wittig D, Oakeley EJ, Haase M, Lam WL and Schübeler D: Chromosome-wide and promoter-specific analyses identify sites of differential DNA methylation in normal and transformed human cells. Nat Genet 37: 853-862, 2005.

25. Kagan J, Srivastava S, Barker PE, Belinsky SA and Cairns P: Towards Clinical Application of Methylated DNA Sequences as Cancer Biomarkers: A Joint NCI's EDRN and NIST Workshop on Standards, Methods, Assays, Reagents and Tools. Cancer Res 67: 4545-4549, 2007.

26. Paci M, Maramotti S, Bellesia E, Formisano D, Albertazzi L, Ricchetti T, Ferrari G, Annessi V, Lasagni D, Carbonelli C, et al: Circulating plasma DNA as diagnostic biomarker in non-small cell lung cancer. Lung Cancer 64: 92-97, 2009.

27. Vlassenbroeck I, Califice S, Diserens AC, Migliavacca E, Straub J, Di Stefano I, Moreau F, Hamou MF, Renard I, Delorenzi M, et al: Validation of real-time methylation-specific PCR to determine O6-methylguanine-DNA methyltransferase gene promoter methylation in glioma. J Mol Diagn 10: 332-337, 2008.

28. Liu BL, Cheng JX, Zhang W, Zhang X, Wang R, Lin H, Huo JL and Cheng $\mathrm{H}$ : Quantitative detection of multiple gene promoter hypermethylation in tumor tissue, serum, and cerebrospinal fluid predicts prognosis of malignant gliomas. Neuro Oncol 12: $540-548,2010$ 